\title{
Evénements en ville, événements de ville : vers de nouvelles ritualités urbaines ?
}

\section{Emmanuelle Lallement}

\section{(2) OpenEdition}

12 Journals

Édition électronique

URL : http://journals.openedition.org/communicationorganisation/275

DOI : 10.4000/communicationorganisation.275

ISSN : $1775-3546$

Éditeur

Presses universitaires de Bordeaux

Édition imprimée

Date de publication : 1 décembre 2007

Pagination : 26-38

ISSN : 1168-5549

\section{Référence électronique}

Emmanuelle Lallement, "Evénements en ville, événements de ville : vers de nouvelles ritualités urbaines? », Communication et organisation [En ligne], 32 | 2007, mis en ligne le 01 décembre 2010, consulté le 19 avril 2019. URL : http://journals.openedition.org/communicationorganisation/275 ; DOI : 10.4000/communicationorganisation.275 
Dossier : La ville dans tous les sens

\title{
Résumé
}

Paris est la scène, depuis quelques années, d'opérations dites « populaires et conviviales » qui jouent sur le principe de rendre accessible des lieux fermés et/ou interdits et de détourner des espaces de leur vocation première, à l'image de Paris Plage, Nuits Blanches, Fête de la Musique, etc. Ces événements festifs urbains offrent à l'ethnologue un terrain d'observation privilégié des modalités de production de la ville aujourd'hui. Le caractère classiquement rituel de ce type de manifestations se donne à voir dans leur dispositif événementiel et scénographique, leur répétition et leur codification. Mais leur ressort symbolique semble jouer également sur le décalage, voire l'inversion des lieux et des comportements associés. C'est la logique de la performance qui semble alors centrale. Comme si un nouveau régime de mise en présence de la ville, Paris en particulier et la grande ville en général, était entrain d'émerger.

\section{Mots-clés}

Paris, ville, fête, événement, rituel, performance, ethnologie.

\begin{abstract}
For several years Paris has been the arena for certain "popular and convivial " events based on the idea of granting access to places previously closed or forbidden to the public, as well as diverting them from their primary function, such as Paris Plage, Nuits Blanches, Fête de la Musique and so on. These urban festivals offer the ethnologist an exceptional terrain to observe the mechanisms of production of the modern city. The classically ritual character of these types of events is evident from the publicity and scenographic devices they employ, as well as their repetition and codification. But their symbolic function also stems from the discrepancy between, or inversion of, the place and the utilisation commonly associated with it. Thus, the logic of performance seems to become central. As if a new system of the presence of the city - Paris in particular and the city in general - was emerging.
\end{abstract}

\section{Key-Words}

Paris, city, festive events, ritual, performance, social anthropology.

\section{Emmanuelle Lallement}

Ethnologue, Maître de conférences à l'Université Paris-Sorbonne, Celsa. Membre du GRIPIC au Celsa et associée au LAIOS/ CNRS/EHESS. Ses recherches portent sur la fabrication du monde urbain. Elle mène des enquêtes sur les événements festifs urbains, sur les situations d'échange marchand et les liens entre commerces et villes, ainsi que sur l'habitat et ses objets. Elle s'intéresse également aux relations interculturelles. 


\title{
Evénements en ville, événements de ville : vers de nouvelles ritualités urbaines ? Emmanuelle Lallement
}

\author{
emmanuelle.lallement@celsa.paris-sorbonne.fr
}

La Mairie de Paris les prépare de longue date, les medias en font largement écho, les Parisiens y participent en nombre. Ces opérations sont devenues au fil des années des rendez-vous qui scandent le calendrier parisien. Mais qu'y a-t-il de commun entre Paris Plage, les Nuits Blanches, la Fête de la Musique, peut-être même encore d'autres événements culturels et festifs urbains ? Paris devient en effet la scène, depuis quelques années, d'opérations festives dites « populaires et conviviales » qui jouent sur le principe de rendre accessible des lieux fermés et/ou interdits et de détourner des espaces de leur vocation ou de leur fonctionnalité première pour en faire, le temps d'une soirée, d'une nuit, d'un été, autre chose que ce qu'ils sont, quelquefois même précisément ce qu'ils ne peuvent pas être ordinairement. Ainsi, la rue devient lieu de concert amateur, les Pompes Funèbres sont transformées en lieu d'exposition d'art contemporain, les quais de la Seine se muent en plage... Peut-on dire que ces manifestations proposent une écriture particulière de la ville ? Peuvent-elles être considérées comme de nouvelles écritures de la ville? Cet article se propose de travailler l'hypothèse selon laquelle ces opérations, de plus en plus nombreuses et récurrentes, à la fois événementielles et bien ancrées dans la vie des citadins, participent des processus de production de la ville aujourd'hui, selon des modalités qu'il s'agit, pour l'anthropologue, de décrire.

Paris Plage est l'opération estivale-phare de la capitale française depuis le début de la mandature du socialiste Bertrand Delanoë à la Mairie de Paris. Avec son service de la communication, le nouveau maire de Paris charge en 2002 une agence de jeunes scénographes ${ }^{1}$ de faire des voies sur berge, autrement appelées «voie Georges Pompidou » du nom du président qui a ouvert en 1967 cet espace à la circulation automobile alors conquérante, une «plage urbaine $»^{2}$. En

\footnotetext{
${ }^{1}$ Jean-Marc Choblet et l'équipe de l'agence de création « Nez Haut».

${ }^{2}$ Depuis, le concept de «plage urbaine» est déposé. Voir le site : www. plagesurbaines.com dans lequel Jean-Christophe Choblet et Sylvie Del Percio de l'agence Nez Haut définissent leur concept qui repose «sur l'éphémère dans l'espace public, la scénographie urbaine et l'évènementiel populaire. Cette expérience de l'éphémère nous pousse à explorer le champ urbain et plus largement l'aménagement de l'espace public par le biais de la scénographie comme outils d'expérimentation. Ainsi, le public
} 
lieu et place de la «deux voies», pour une période donnée qui correspond au cœur de l'été, les services municipaux installent désormais chaque année du sable, des palmiers, des pelouses, des transats, des guinguettes, un mur d'escalade, une piscine, une zone de brumisateurs géants, un labyrinthe d'eau, des trampolines, un terrain de pétanque... Le maire en a fait une opération emblématique de sa politique municipale en affirmant qu'il s'agit de « rendre aux Parisiens les berges de leur fleuve» et ceci «dans un esprit populaire, festif, civique et convivial $»^{3}$. Lors des conférences de presse qui précèdent chaque nouvelle édition, les services de communication de la mairie de Paris annoncent que Paris Plage est un espace de détente pour tous les Parisiens, mais aussi les habitants de la banlieue et les touristes. Depuis sa création, si tant est que les conditions météorologiques le permettent, près de deux millions de visiteurs participent chaque été à Paris Plage, les uns pour flâner, les autres pour " voir le phénomène » et le commenter, d'autres encore pour bronzer, pour lire, pour jouer à la pétanque, pour pique niquer, pour écouter de la musique ${ }^{4} \ldots$

La Fête de la musique, quant à elle, existe depuis bien plus longtemps. Avant d'être cette fête célébrée dans un grand nombre de pays du monde à une même date, le 21 juin, marquant le solstice d'été comme autrefois la St Jean, elle a été une opération plus locale, lancée en 1982 par Jack Lang alors ministre de la Culture du premier gouvernement de François Mitterrand. Partant du constat que cinq millions de Français pratiquent un instrument, en amateurs ou en professionnels, les organisateurs veulent « faire descendre la musique

est invité à investir différemment son cadre de vie. Si ce rendez-vous citoyen s'avère positif, l'outil ainsi créé, légitime le choix des décideurs de pérenniser ou non ces aménagements éphémères. »

${ }^{3}$ Discours de Bertrand Delanoë à l'occasion de la conférence de presse de juillet 2003.

${ }^{4}$ Voir Emmanuelle Lallement «Célébrer un objet absent. L'opération Paris Plage » dans Objets et mémoires, sous la direction d'Octave Debary et Lauier Turgeon, MSH/Celat, Paris, Laval, 2077.

5 « En moins de 15 ans, la Fête de la Musique sera reprise dans plus de 100 pays. Si sa dimension européenne reste la plus visible, maintenant que Berlin, Budapest, Barcelone, Istanbul, Liverpool, Luxembourg, Rome, Naples, Prague, la Communauté française de Belgique, Santa Maria da Feira... ont signé une "Charte des partenaires de la Fête européenne de la Musique", la Fête s'est développée à San Francisco, à NewYork, à Manille, et est pratiquement devenue fête nationale dans de nombreux pays du continent africain, sans parler du Brésil ou de la Colombie». source : Fetedelamusique.culture.fr (site officiel de la Fête de la musique). 
dans la rue $»^{6}$ selon la formule : «la musique partout et le concert nulle part ». Chacun est donc invité à investir la rue pour jouer et/ou écouter de la musique, le jeu de mot «Faites de la musique, Fête de la Musique » en étant le slogan fondateur. La réussite est telle que la Fête de la Musique devient très vite, tout en conservant l'image d'une manifestation populaire et spontanée, une institution, à Paris comme dans toutes les villes françaises, et depuis quelques années, à l'étranger également.

Les Nuits Blanches constituent une autre opération festive montée elle aussi par la Mairie de Paris. "En 2002, Christophe Girard, maire adjoint chargé de la culture, propose à Bertrand Delanoë, maire de Paris, de créer un parcours artistique nocturne dévolu à l'art contemporain durant toute une nuit à Paris. Rendre l'art accessible à tous, mettre en valeur l'espace urbain par la création moderne, créer un moment de convivialité : tels sont les enjeux fixés pour cette nouvelle manifestation ${ }^{7}$. Régulièrement confiée à Jean Blaise, le créateur d'un autre événement urbain, les Allumés de Nantes, l'organisation consiste chaque année à identifier un ensemble de lieux de la ville, habituellement destinés à l'art comme le Palais de Tokyo, le Petit Palais, les galeries d'art du Marais ou bien des lieux plus insolites et décalés comme les Pompes Funèbres, les jardins de l'hôpital de la Pitié Salpêtrière, la place Sainte Marthe, les abords du métro Belleville ou l'Eglise Saint Jean de Montmartre, dans lesquels des artistes contemporains sont invités à présenter des installations.

D'autres manifestations culturelles (nationales, voire européennes) paraissent relever de la même volonté de proposer à date fixe, chaque année, de rendre accessible ce qui ne l'est pas a priori, ou de révéler sous un nouveau jour des lieux ou des œuvres méconnues. Les journées du Patrimoine, créées en 1984, permettent aux Français et aux touristes, chaque troisième week-end de septembre, d'entrer à l'Elysée, de découvrir selon un autre usage des bâtiments publics (Bourse du Travail, Hôpital Lariboisière, Mairies d'arrondissement, Hôtel de Ville...), de visiter gratuitement des musées à l'entrée habituellement payante, d'écouter des conférences sur des thèmes historiques (les 200 ans du Code du commerce, l'héraldique, l'histoire

\footnotetext{
${ }^{6}$ Mais les ethnologues ont montré que la tradition de la musique de rue est bien plus ancienne. Voir « Musiques dans la rue ». Sous la direction d'Éliane Daphy et Florence Gétreau. Ethnologie française, XXIX, 1999/1.

Voir également Musiciens des rues de Paris, Sous la direction de Florence Gétreau. Paris, Réunion des musées nationaux, 1997.

${ }^{7}$ Site officiel des Nuits Blanches sur www.paris.fr/culture
} 
de la ville de Paris...). «Parallèlement aux chefs d'œuvre de l'architecture civile ou religieuse sont mis à l'honneur les témoins des activités industrielles ou agricoles, les parcs et jardins, les sites archéologiques, les objets mobiliers, le patrimoine littéraire, fluvial ou militaire... $>^{8}$. Quant à la Nuit des Musées, dont la troisième manifestation a eu lieu en 2007 dans toute la France et en Europe, elle consiste à ouvrir des institutions muséales, durant toute une nuit au printemps, afin de "célébrer de manière insolite et ludique les richesses méconnues de notre patrimoine muséale $»^{9}$.

Certes toutes ces opérations ne sont pas semblables et ont chacune leur histoire, leur lien avec les politiques culturelles, leurs conditions d'élaboration et de développement et leurs participants. Mais on ne peut ignorer en revanche que ces manifestations sont de plus en plus fréquentes et que le nombre de villes concernées par ce type d'événements est toujours plus grand. Beaucoup de villes, en France et ailleurs, organisent des événements qui mêlent ambitions culturelles (la culture contemporaine ou le patrimoine culturel accessibles), politiques publiques urbaines (requalification des espaces urbains existants, renouvellement urbain) et objectifs dits sociaux (lien social, convivialité, mélange...). Egalement ces opérations apparaissent comme des succès du point de vue de la fréquentation. Comptabilité est faite et reprise dans la presse du nombre de visiteurs, toujours plus important (pour exemple Nuits Blanches est passé de 500000 visiteurs la première année à 2 millions en 2006), de la quantité de lieux ouverts, du temps passé dans les files d'attente... Enfin, ces événements ont tous connu une expansion fulgurante : la Fête de la Musique a été organisée dès la deuxième année dans toutes les villes de France et même au delà, Paris Plage a été copié immédiatement en province et à l'étranger, les Nuits Blanches parisiennes ont été rapidement exportées à Rome. Il est donc possible de faire le pari que toutes ces opérations ont quelque chose en commun, qu'elles relèvent d'une même logique qu'il s'agit de mettre au jour.

\section{Aujourd'hui comme hier, des fêtes et des rituels...}

A première vue, rien de bien nouveau sous le ciel de l'anthropologie dans ces événements festifs urbains. Les villes d'aujourd'hui, comme celles de jadis, mais aussi comme les villages, connaitraient toutes leurs fêtes, traditionnellement qualifiées de rituelles. Les carnavals, les charivaris, les nuits de la Saint Jean, ont en effet constitué des

\footnotetext{
${ }^{8}$ Site officiel des Journées du Patrimoine sur www.journeesdupatrimoine.culture.fr

${ }^{9}$ Site officiel de la Nuit des Musées sur nuitsdesmusees.culture.fr
} 
phénomènes centraux dans l'analyse des rites populaires. Durkheim voit dans la fête rituelle des moments d'effervescence collective qui expriment des réalités collectives. Plus tard, Jean Duvignaud y repère déjà les composants indispensables au dérèglement savamment réglé des fêtes urbaines : «La rue, les cours, les places, tout est bon pour cette rencontre des hommes en dehors de leurs conditions et du rôle qu'ils jouent dans une société organisée $»^{10}$.

Les villes d'aujourd'hui n'échapperaient donc pas à la logique symbolique qui anime ce type de pratique sociale: ces événements, qu'ils soient des fêtes ou des événements culturels, relèveraient de la même nécessité pour le groupe social de se réaffirmer en décidant d'un temps, d'un espace et d'une activité à la fois très codifiée et décalée par rapport à la vie ordinaire. Plusieurs caractéristiques de l'activité rituelle permettent en effet de décrire aussi bien Paris Plage que les Nuits Blanches ou même la Fête de la musique.

La première caractéristique concerne la forme événementielle mais répétitive de certaines opérations festives. Nuits Blanches, Paris Plage, Fête de la musique, semblent à la fois jouer sur le registre exceptionnel tout en étant attendues à une date précise chaque année. Il ne s'agit pas en effet de l'événement one shot de la Coupe du Monde de football, qui a en tant que tel été perçu comme «à nul autre pareil $»^{11}$, qui reste une référence en matière de manifestation populaire "spontanée » et à laquelle on fait appel pour mesurer le succès d'autres manifestations de liesse. Si Paris Plage ou Nuits Blanches sont des événements, ils confèrent plutôt ici au rendez-vous. Ils jouent à la fois sur le fait de produire une rupture par rapport au cours ordinaire des espaces et du rythme urbain, tout en se réitérant de manière régulière, attendue, et immanquablement commentée par la presse. C'est donc en quelque sorte à leur résurrection que les citadins assistent chaque année. Ne parle-t-on pas d'ailleurs, précisément pour désigner le retour de telle ou telle opération, de sa «nouvelle édition »? On crée ainsi une sorte de ritualité urbaine. Et c'est ce qui constitue, en quelque sorte, l'événement. Ces fêtes sont également associées à des temps bien précis, qui en marquent la saisonnalité : à l'automne ses Nuits Blanches, en plein été son Paris Plage, à l'arrivée de l'été sa fête de la Musique. La dimension temporelle des fêtes a été régulièrement au centre des analyses des folkloristes qui voyaient par

\footnotetext{
${ }^{10}$ Fêtes et civilisations, Actes Sud, Paris, 1991, p. 49.

${ }^{11}$ Pour une approche anthropologique de la notion d'événement, voir «Qu'est-ce qu'un événement?», Terrain $\mathrm{n}^{\circ} 38$, mars 2002. Voir en particulier A. Bensa et E. Fassin « Les sciences sociales face à l'événement » pp. 5-20.
} 
exemple dans le carnaval un moment fort parmi les fêtes de l'hiver à relier avec le Carême ${ }^{12}$.

La deuxième analogie possible est celle du caractère codifié de ce type d'événements. A Paris Plage comme pour les Nuits Blanches, les participants suivent un parcours, en apparence libre mais clairement identifié. Un parcours qui ordonne le réel de la ville d'une certaine manière. Le promeneur est guidé pas à pas dans une ville faite de lieux reconnus selon de nouveaux codes (accessibilité, gratuité, nouvelle mise en scène), engendrant de nouveaux usages. Et dans chaque lieu, la codification est centrale : l'idée de ces dispositifs est de déclencher des actions et que ces actions soient contenues dans le dispositif prévu à cet effet. On fait la sieste dans les transats et on joue à la pétanque dans le terrain balisé pour ce qui concerne Paris Plage, on ne sort son saxophone que le soir de la Fête de la Musique et on suit le sens de la circulation autour de l'installation d'un artiste contemporain pour les Nuits Blanches. Si d'ordinaire l'événement produit du rituel, engendre une manière rituelle de le vivre, dans le cas de Paris Plage, le rituel semble résider dans l'événement lui-même, comme s'il était prévu $a$ priori, en quelque sorte décrété. Sa scénographie propose aux visiteurs de suivre un parcours type, symbolisé comme une "journée à la plage », depuis le lever jusqu' au coucher du soleil. Partant du Pont des Arts, le parcours, jalonné par des repères comme un coin taï chi, un stand de dessins, ou une aire de pelouse avec ses transats, aboutit à Sully Morland où, en fin de journée, le visiteur est invité à siroter un verre dans un bar guinguette et à jouer à la pétanque dans un espace dédié. Le rituel est pensé dès la conception des projets. Et l'objectif de ce type d'opérations est de déclencher des comportements qui, d'une certaine manière, font des clins d'œil au caractère ritualisé ${ }^{13}$ d'un certain nombre de pratiques ordinaires.

La troisième caractéristique permet de qualifier le type de rituel mis en place dans ces opérations festives urbaines. On peut en effet s'interroger sur le point commun de tous ces événements, à savoir le détournement, voire l'inversion, matérielle et symbolique, qu'ils opèrent de la ville et des comportements qui y sont associés. "Les

\footnotetext{
${ }^{12}$ Voir Julio Caro Baroja, Le Carnaval, NRF Gallimard, Paris, 1979.

13 «Toutes les virtualités offertes aux praticiens ordinaires de la vie quotidienne composent un ensemble défini d'activités indéfiniment ritualisées. Le citoyen moyen, en ce sens citoyen modèle, s'entend, comme père de famille, travailleur, joueur de tiercé, amateur de sport, automobiliste, à mettre en œuvre et pratiquer le rite. Il fait ce qu'il faut quand il faut et se présente à l'heure dite au bureau ou à l'usine, aux urnes, au stade, aux portes de Paris et à Merlin-Plage. ", Marc Augé dans Pouvoirs de vie, pouvoirs de mort, Flammarion, Paris, 1977, p. 8.
} 
historiens, les sociologues et les géographes s'accordent à trouver dans les fêtes rurales et urbaines, religieuses ou non, certains ingrédients communs: l'excès, la parodie, la transgression, la fantaisie, la ferveur, la joie. Mais ce qu'ils constatent dans les fêtes contemporaines - dont on ignore bien souvent l'origine - c'est une sorte de reconquête, pour chacun et pour tous, du temps et de l'espace. Il n'y a plus d'horaire à respecter, le temps est entièrement libre, disponible, on peut s'attarder ici, paresser là, rien ne presse... Il n'y a pas, non plus, de territoires interdits ou confisqués, toute la ville est ouverte et offerte. Même les automobilistes la jouent discrète... Certains rêvent alors d'une ville en fête ininterrompue sans comprendre que c'est justement l'exception qui confirme la règle ! ». ${ }^{14}$ Ne s'agirait pas de mettre en commun un «autrement» de la ville et de ses repères habituels? Un « autrement» qui prendrait la forme de la « ville autre » et qui s'incarnerait dans des lieux, dans des moments et dans des rassemblements inédits ?

Notons que c'est la nuit que certaines de ces opérations prennent place, le caractère nocturne signifiant la dimension festive, voire transgressive ainsi promise. L'expression «c'est magique ! » revenant avec récurrence dans les commentaires des spectateurs des installations des Nuits Blanches ou des visiteurs des lieux rendus accessibles. La ville dévoilerait la nuit, aux citadins qui le désirent, ses coulisses, et s'offrirait d'une nouvelle manière. Ouvrir la nuit les salles du Musée de la Chasse une seule fois par an constitue un événement, en comparaison duquel les nocturnes hebdomadaires du musée du Louvre n'ont plus rien d'exceptionnel. Et en flânant dans les rues de sa ville la nuit, c'est à un citadin libéré de ses contraintes auquel on jouerait. La nuit autoriserait un autre rythme que celui du quotidien diurne, ainsi qu'une meilleure disposition des participants.

Ensuite, toutes ces manifestations ont en commun d'être gratuites, ce qui est une manière de détourner la logique habituelle de certains lieux. Non seulement les lieux de la ville sont ouverts alors qu'ils devraient être fermés mais l'accès y est libre et gratuit. La rupture avec les logiques qui régissent l'ordinaire des rapports des citadins avec leur ville se joue donc également dans cette mise en scène de l'accessibilité et du caractère offert des lieux. Pour quel type d'activité et d'usage de la ville de tels rassemblements ont lieu ? Là encore, c'est au versant inhabituel des pratiques que ce type d'événements autorise : le citadin n'est pas dans la posture du consommateur, de

14 Thierry Paquot, introduction à «Fête la ville ! », Urbanisme $\mathrm{n}^{\circ} 331$, juillet-août 2003. 
l'habitant qui fait ses courses, il n'est pas non plus l'usager des transports en commun.

La géographie parisienne est aussi soudain bouleversée pour chacune de ces manifestations. La ballade, la déambulation, la flânerie viennent rompre avec l'habitude du trajet quotidien. La ville devient lors des Nuits Blanches un territoire à parcourir à pieds ou à vélo et non plus en voiture ou en transports en commun.

Si la Fête de la Musique, Paris Plage et les Nuits Blanches proposent une lecture particulière de la ville, c'est parce qu'ils auraient une écriture particulière de la ville, qui fonctionne sur le principe de la pirouette, du décalage. La Fête de la Musique, en tant qu'événement qui permet de voir fleurir, à chaque coin de rue, sur toutes les places et dans des espaces variés et quelquefois insolites, des concerts amateurs et professionnels, transfigure l'espace public parisien. Le Palais Royal devient lieu de concert, la place de la Bastille se transforme en bal géant, le parvis de l'Institut du Monde Arabe accueille des groupes de musiciens dits « du monde». De son côté, Paris Plage prend place dans un lieu ordinairement dédié au trafic automobile, dans un espace qui traverse la ville mais qui n'appartient à aucun quartier en particulier. Les quais de la Seine, à la fois cœur de Paris mais échappant à la logique des quartiers, tout en étant site classé patrimoine mondial par l'Unesco, figurent un espace qui, à la manière d'une friche, peut être investi selon un mode inédit, ici la plage dans la ville. Les Nuits Blanches, quant à elles, dessinent une géographie spécifique de la ville, qui s'accompagne d'ailleurs d'un plan de Paris conçu pour l'occasion, quartier par quartier. On choisit de « faire les Batignolles » ou de « rester dans le Marais ». Mais on peut tout aussi bien se retrouver dans un nouveau quartier, érigé pour l'occasion en espace emblématique de la ville, «Tolbiac les Olympiades » ou bien le «quartier Carpentier». Lieux historiques de la capitale se retrouvent donc pour les Nuits Blanches associés à des quartiers plus méconnus et c'est la logique d'ensemble ainsi formée qui, provoquant une certaine nouveauté, participe à produire l'événement. Egalement le décalage est à l'honneur dans chacun des lieux investis pour l'occasion. L'effet des installations artistiques est en effet de déclencher un certain étonnement.

Ces manifestations fonctionnent donc sur le principe soit du off, révéler des coulisses, soit de l'exhibition d'un lieu sous un autre jour, selon une nouvelle lumière. Les installations artistiques sous forme de mise en lumière sont d'ailleurs assez nombreuses lors des Nuits Blanches. C'est la logique de l'insolite qui semble primer, un insolite 
qui se trouve dans l'ordinaire comme dans l'exceptionnel. On peut donc tout aussi bien découvrir un quartier qu'on ne fréquente jamais que redécouvrir son propre quartier pour l'occasion transfiguré. Pour les Journées du Patrimoine, les villes en général, et Paris en l'occurrence, deviennent un terrain de découvertes de patrimoines institués tout aussi bien qu'insoupçonnés. Paris se révèle alors comme la somme des lieux méconnus ici mis en valeur, ou franchement inconnus et tout à coup mis en vedette, ou bien encore comme des espaces habituellement fermés au grand public et tout à coup accessibles ou enfin comme des lieux ordinaires qui se transforment en installation.

Le caractère événementiel de ce type d'opérations semble enfin jouer sur une rupture d'ordre social, à savoir le rassemblement inhabituel d'individus aux origines sociales, culturelles, voire ethniques variées qui d'ordinaire ne se côtoieraient pas dans les mêmes lieux urbains. Dans une atmosphère souvent qualifiée de conviviale, voire fraternelle, des rapports sociaux en rupture avec les rapports ordinaires de hiérarchie, de domination, de ségrégation seraient établis. Bref c'est la situation sociale, socialement créée, qui est inédite et qui, en tant que telle, met en scène l'inversion dans le rituel.

\section{La performance comme nouvelle ritualité}

Néanmoins, comment expliquer que des milliers de Parisiens « jouent» à aller à la plage chaque été en plein Paris ? Comment comprendre que des millions de gens à travers le monde s'improvisent musiciens et/ou mélomanes dans chaque recoin de leur ville et acceptent de ne plus les investir le jour suivant? Que voir dans ce phénomène de masse qui consiste à patienter dès l'aube devant l'Elysée ou un autre bâtiment officiel ordinairement fermé au public ? Certes il s'agit de faire ce qui n'est pas autorisé habituellement, de conquérir ou reconquérir ce qui est réservé à d'autres, de transgresser en quelque sorte. Mais personne n'est dupe, le jeu consiste, là comme ailleurs, à accepter l'illusion, voire la fiction. Les visiteurs sont invités à participer et font partie du processus même de transfiguration des lieux de la ville. Car c'est la dimension performative des pratiques qui est convoquée dans ces situations qui s'apparentent alors à des «moments d'enchantement ${ }^{15}$. Il y a bien dans ces événements festifs urbains une volonté de suspension, dans un temps et un espace

\footnotetext{
${ }^{15}$ Selon la définition d'Yves Winkin dans «Anthropologie de l'enchantement. Du tourisme et d'autres pratiques de suspension » et « Le touriste et son double. Eléments pour une anthropologie de l'enchantement », S. Ossman, éd., Miroirs maghrébins. Itinéraires de soi et paysages de rencontre, Paris, CNRS Editions, 133-143.
} 
codifiés, de l'incrédulité habituelle, et ceci grâce à l'intervention d'artistes ou de concepteurs. Paris Plage en serait l'exemple le plus frappant: la singularité de l'événement repose sur un ressort symbolique qui propose une forme d'inversion particulière puisqu'il fait d'un espace urbain une plage, c'est-à-dire précisément ce que ne peut pas être un espace urbain. Et c'est en effet parce que tout le monde joue à la plage autour de l'absence de l'élément fondateur de la plage, à savoir la mer, que cette opération est un événement et non un simple équipement urbain. Tout le monde semble s'accorder, par jeu et implicitement, sur cette mise en scène. D'ailleurs il ne s'agit pas de faire croire aux citadins qu'ils sont à la mer. La plage ici proposée n'a rien de vraisemblable, tant ses éléments (le sable blanc, les hamacs, les oriflammes, les brumisateurs, la piscine même) sont rassemblés non pas en tant qu'ils sont réels, mais en tant qu'ils constituent des symboles de la plage. En quelque sorte, plus on s'accorde sur la mise en présence de l'absence de la mer, plus on joue le jeu non pas de la plage mais de la performance de la plage.

Cette performance a pour condition, aussi bien que pour enjeu, de faire des participants à ce type de manifestations des citadins-acteurs, indispensables aux dispositifs. Acteurs d'une ville qui ne peut exister que dans sa dimension performante et éphémère, les citadins ainsi « enchantés 》 goûteraient le plaisir du rassemblement, un rassemblement qui ne fait que des égaux pour l'occasion et qui les laisserait dans une indétermination plutôt ludique de leur identité, voire permettrait une sorte de mimicry ${ }^{16}$. A Paris Plage ou aux Nuits Blanches, aux Nuits des musées et aux Journées du Patrimoine, le jeu n'est certes pas celui du masque et du costume, mais en s'accordant tous pour détourner un lieu, sa vocation ou sa fonction, les participants se transforment en performers, par conséquent d'une certaine manière auteurs d'une translation.

La logique de la performance a également pour effet de faire advenir une nouvelle ville. Les dispositifs festifs éphémères parisiens jouent sur des opérations de détournement de Paris, qui peuvent être vus comme des mises en présence d'un certain Paris, par des dynamiques

\footnotetext{
${ }^{16}$ Roger Caillois définit ainsi une des dimensions possibles du jeu : «Tout jeu suppose l'acceptation temporaire, sinon d'une illusion (...), du moins d'un univers clos, conventionnel et, à certains égards, fictif. Le jeu peut consister, non pas à déployer une activité ou à subir un destin dans un milieu imaginaire, mais à devenir soi-même un personnage illusoire et à se conduire en conséquence. On se trouve alors en face d'une série variée de manifestations qui ont pour caractère commun de reposer sur le fait que le sujet joue à croire, à se faire croire ou à faire croire aux autres qu'il est un autre que lui-même. » Les jeux et les hommes, Paris, Idées/Gallimard, 1967.
} 
de mise en absence de ce qu'est ordinairement la ville, de suspension esthétique de la ville. On serait alors bien dans la logique de l'installation : un rendez-vous, dans un espace donné, pour conférer à un espace un autre statut. Il s'agit de proposer un nouveau régime de présence de certains lieux de la ville, qui ainsi sortent de leur banale fonctionnalité pour acquérir un statut particulier, celui de matière ou de support dont on peut faire une œuvre. Paris est, lors des Nuits Blanches, jalonné d'installations, de performances, d'œuvres éphémères. Paris Plage, dont les scénographes ont pris soin de faire protéger juridiquement le concept, transforment de manière éphémère les quelques kilomètres de voies de circulation en une fiction, celle de la plage en plein Paris. Si bien que c'est la ville elle-même qui devient, en quelque sorte, une performance, une œuvre. Comme si un nouveau régime de mise en présence de la ville, par ce qu'elle ne peut pas être, émergeait.

Enfin, une telle analyse invite à s'interroger sur le phénomène de répétition et de copie de ce type d'événements urbains. Comment produire quelque chose d'unique, de l'ordre de l'œuvre, quand celle-ci peut être copiée, ici et ailleurs ? On le sait, la Fête de la musique, Paris Plage, les Nuits Blanches, sont à la fois typiquement parisiens mais se sont exportés avec succès, en France comme à l'étranger. Qu'est-ce qui est copié dans ces Paris Plage toulousains, lyonnais, hongrois (Buda Plage) ? Qu'est-ce qui est repris des Nuits Blanches de Paris quand elles deviennent les Notte Bianca de Rome? Quel genre d' « air de Paris » souffle dans ces « copier-coller» de la ville ? Est-ce Paris en tant que ville singulière, unique? Mais n'est-ce pas aussi, conjointement, par la logique de la performance ritualisée, l'urbain en général, à ce titre reproductible à l'envi, au-delà des particularités locales ? A-t-on alors affaire ici à une écriture transposable à toutes les grandes villes du monde, faisant ainsi de cette nouvelle ritualité urbaine une marque de fabrique de la modernité urbaine en général ? Tous ces événements, largement médiatisés en France et à l'étranger, semblent en effet participer de l'image que veut se donner la ville de Paris aujourd'hui, à savoir une capitale de rang mondial qui, comme New York ou Londres, a ses grands événements festifs et culturels, sans pour autant cesser de jouer la carte de la ville conviviale et à l'esprit «bon enfant». La ville ainsi produite est alors une sorte de concept que l'on peut exporter, vendre, communiquer. Le lien entre fête et ville semble alors prendre un tour nouveau. Comme le souligne André Sauvage «La fête a quelque chose à voir avec la polis, avec le et la politique, par le simple fait qu'elle semble avoir définitivement 
rallié la ville ${ }^{17}$. Ces événements festifs urbains traduisent la volonté de production d'une identité locale singulière, celle de Paris, qui serait ainsi différente de celle de Rome, Madrid ou San Francisco, donc indispensable sur le marché concurrentiel des identités de ville. Mais ce type de manifestations, assez semblables de ville de ville, reproduites ici et ailleurs et répétées d'année en année, tend également à produire une identité urbaine plus générique, qui définit ainsi la « marque » de la grande ville. C'est sans doute cette double logique qui permet de conserver, dans la copie comme dans la répétition, la dimension événementielle de ces opérations.

\section{Bibliographie}

Caillois R., Les jeux et les hommes, Paris, Idées/Gallimard, 1967.

Duvignaud J., Fêtes et civilisations, Paris, Actes Sud, 1991.

Lallement E., De La Pradelle M., «Célébrer un objet absent. L'opération Paris Plage », in : Objets et mémoires, MSH/Celat, Paris, Editions Laval, 2007.

Lallement E., De La Pradelle M., «On the waterfront. Paris Plage : the city is ours ", in The annals of the American Academy of Political and Social Sciences, Sage Publications, Thousand Oaks, CA, USA, septembre 2004.

Millet C., L'art contemporain, Paris, Flammarion, 1997.

\footnotetext{
${ }^{17}$ «Fête la ville ! », Urbanisme $\mathrm{n}^{\circ}$ 331, juillet-août 2003.
} 
